\title{
Detection of TNT using a sensitive two-photon organic dendrimer for remote sensing
}

\author{
Aditya Narayanan ${ }^{1}$, Oleg Varnavski ${ }^{1}$, Oliver Mongin ${ }^{2}$, \\ Jean-Pierre Majoral ${ }^{3}$, Mireille Blanchard-Desce ${ }^{2}$ and \\ Theodore Goodson III ${ }^{1,4}$ \\ ${ }^{1}$ Department of Chemistry and Macromolecular Science and Engineering, NSF Center for \\ Ultra-fast Optical Science (FOCUS), University of Michigan, Ann Arbor, MI 48109, USA \\ ${ }^{2}$ Synthèse et ElectroSynthèse Organiques (CNRS, UMR 6510), Université de Rennes 1, \\ Institut de Chimie, Campus Scientifique de Beaulieu, Bât 10A, F-35042 Rennes Cedex, France \\ ${ }^{3}$ Laboratoire de Chimie de Coordination, CNRS, 205 route de Narbonne, F-31077, \\ Toulouse Cedex 4, France \\ E-mail: tgoodson@umich.edu
}

Received 26 November 2007, in final form 14 January 2008

Published 18 February 2008

Online at stacks.iop.org/Nano/19/115502

\begin{abstract}
There is currently a need for superior stand-off detection schemes for protection against explosive weapons of mass destruction. Fluorescence detection at small distances from the target has proven to be attractive. A novel unexplored route in fluorescence chemical sensing that utilizes the exceptional spectroscopic capabilities of nonlinear optical methods is two-photon excited fluorescence. This approach utilizes infra-red light for excitation of remote sensors. Infra-red light suffers less scattering in porous materials which is beneficial for vapor sensing and has greater depth of penetration through the atmosphere, and there are fewer concerns regarding eye safety in remote detection schemes. We demonstrate this method using a novel dendritic system which possesses both excellent fluorescence sensitivity to the presence of TNT with infra-red pulses of light and high two-photon absorption (TPA) response. This illustrates the use of TPA for potential stand-off detection of energetic materials in the infra-red spectral regions in a highly two-photon responsive dendrimer.
\end{abstract}

(Some figures in this article are in colour only in the electronic version)

\section{Introduction}

Chemical sensors for nitro-aromatics offer new approaches to the rapid detection of ultra-trace analytes from high explosives [1-4]. This is of vital importance for a broad range of applications from national security to the clean up of mine fields and demilitarized installations. Organic conjugated polymers have proven very useful in creating highly sensitive fluorescent chemical and biological sensors [1-10]. Swager et al [3, 4, 7, 8] have demonstrated high sensitivity of the fluorescence of properly designed polymers to nitro-

4 Address for correspondence: Department of Chemistry, University of Michigan, Ann Arbor, MI 48109, USA. aromatics with the capability to detect the vapors of TNT and other related compounds. Recently non-polymeric materials which are capable to sense TNT vapors such as porphyrin-doped mesoporous silica film [9] or fluorescent nanofibril film [10] have also been demonstrated. Previous work on sensory applications utilized one-photon resonance fluorescence excitation $[4,7,8]$ which implies the use the visible light for the fluorescence excitation. This method when applied for remote sensing can impose certain limitations regarding scatter, absorption in the atmosphere and concerns regarding eye safety for explosives detection.

Functionality of the fluorescence sensors can be greatly increased by the use of a two-photon fluorescence excitation scheme using infra-red light to overcome these limitations. In 
(a)

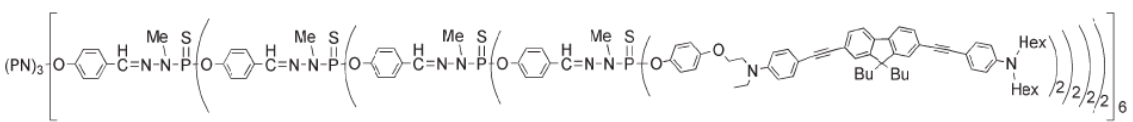

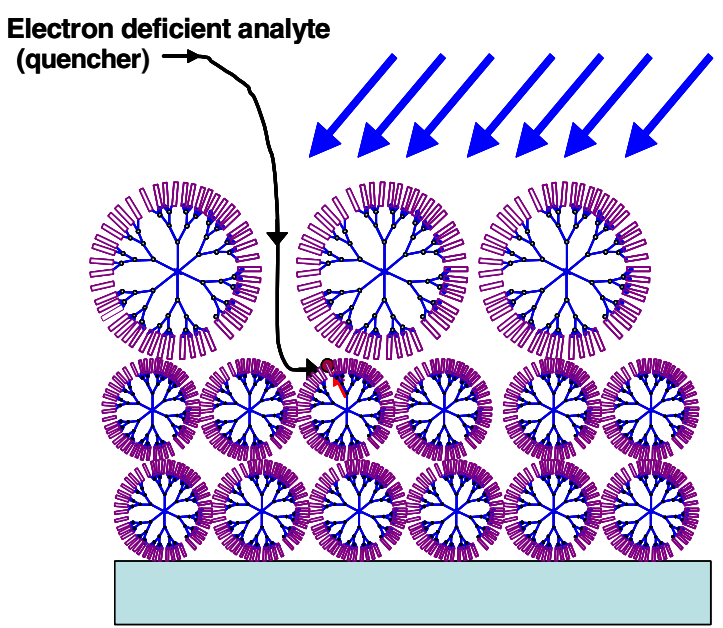

Figure 1. Dendrimer architecture: (a) generation 4 with 96 units shown; (b) spherical dendrimer molecules of various diameters (generations) are prospective for making materials with controllable porosity and enhanced surface area. IR pulses excite the dendritic system which has a porous nature allowing excellent diffusion of the analyte to the sensing units.

order to utilize this technique it is necessary to synthesize novel materials with excellent two-photon absorption properties, and that the same time, demonstrate exceptional sensitivity to the presence of energetic nitro-aromatic compounds. The development of conjugated organic molecules with large two-photon absorption cross-sections $\left(\delta_{\mathrm{TPA}}\right)$ has been of interest in many areas of scientific research [11-15]. A remarkable progress has been done in design approaches for the synthesis of organic molecules with large $\delta_{\mathrm{TPA}}[14,15]$ which can exceed 10000 GM for recently developed large macromolecular systems [15]. Our previous investigations of branched organic molecular structures and dendrimers have shown a great deal of promise for large two-photon responses $[14,15]$. A dendrimer's architecture also allows one to concentrate a large amount of TPA-efficient chromophores in an ordered confined geometry. This results in a large $\delta_{\text {TPA }}$ per dendrimer macromolecule thus providing high TPAexcitation efficiency using femtosecond lasers with modest average power.

Many explosives are highly nitrated organic compounds which are essentially electron-deficient. Their electron accepting capability is exploited in the detection schemes by quenching the fluorescence of electron donor sensory system [4]. Electron-rich conjugated molecular systems are promising candidates for such redox sensing. Another important feature which is essential for the sensor is amplified quenching which results from the rapid migration of excitons through the material and the effective 'sampling' of many different receptor sites $[7,8,16]$. This process has been suggested to enhance the probability of finding an occupied binding site by TNT or DNT polymers [7, 8]. However, an efficient exciton transport is not limited to polymers. It has been observed in dendrimers [14] and other nanoscale systems [17]. Nevertheless, investigations of macromolecular architectures other than linear polymers for the fluorescence sensing with amplified quenching by energetic nitro-aromatics have been limited $[9,10]$.

In this contribution we propose the dendrimer architecture for efficient two-photon-excited fluorescence sensors of TNT. The dendrimer's well defined tree-like mono-dispersed structures lead to a number of interesting characteristics such as globular, void-containing shapes [18, 19], good electronic excitation transport properties within the macromolecule $[14,15,18,20]$, and high nonlinear optical responses $[14,15]$. The surface of globular dendrimer can be functionalized with chromophores specifically sensitive to the presence of electron-deficient quenchers such as nitroaromatics. It has been previously shown that porosity of a sensory material plays a crucial role in the sensitivity mechanism to TNT and other energetic materials [8,9]. Due to a high degree of disorder in a polymer film it is very difficult to control the porous structure in this material. The developed porous structure in a dendrimer provides a condition for the efficient diffusion of analytes to sensing elements, while the large surface area enhances the number of interaction sites between analyte molecules and sensing elements [8]. This is schematically shown in figure 1 . 
However porous films demonstrating the strongest sensory effect $[8,9]$ can potentially produce a strong light scattering in the UV-vis region preventing efficient excitation conditions for relatively thick films. A multi-photon excitation route of such porous materials can have much higher penetration depths of the excitation beam and also minimize scattering, when compared with one-photon excitation.

We herein report dendritic systems which combine excellent TNT-sensing properties, with exceptional two-photon properties at relatively low average excitation power. The complete synthesis and characterization of the dendrimers were reported previously [21]. Generations G1-G4 (figure 1) of the dendrimer possess high quantum yields ranging from 0.48 to 0.75 , absorb in the $400 \mathrm{~nm}$ region, and emit in the $430 \mathrm{~nm}$ region [21].

\section{Experimental details}

\subsection{Two-photon excited fluorescence measurements}

The laser used for the study was a Kapteyn Murnane (KM) mode-locked Ti:sapphire laser. The bandwidth at $800 \mathrm{~nm}$ was $\sim 45 \mathrm{~nm}$, and the pulse duration was $\sim 30 \mathrm{fs}$. The input power from the laser was fixed by using a polarizer. An iris was placed prior to the polarizer in order to ensure a circular beam. The beam from the polarizer was focused on the sample cell (quartz cuvette, $0.5 \mathrm{~cm}$ path length) using a lens with a focal length of $11.5 \mathrm{~cm}$. The fluorescence was collected in a direction perpendicular to the incident beam. A 1-inch focal length plano-convex lens was used to direct the collected fluorescence into a monochromator. The output from the monochromator was coupled to a photomultiplier tube. The photons were converted into counts by a photon counting unit. A logarithmic plot between the collected fluorescent photons and input intensity gave a slope of 2 . This gives a quadratic dependence of the fluorescence on the input intensity which indicates a nonlinear optical response, which in our case is twophoton absorption.

\subsection{Fluorescence quenching measurements}

Fluorescence quenching experiments were carried out by micro-titration in solution. The one-photon excited fluorescence and the two-photon excited fluorescence quenching were performed as follows. Initially, the one-photon excited fluorescence spectrum was recorded in the absence of quencher at room temperature, and measurement of the two-photon excited fluorescence spectrum following subsequently. For the two-photon excited fluorescence quenching, we used the same laser and optical parameters described above. The onephoton excited fluorescence quenching was performed using the Fluoromax-2 (SPEX) spectrophotometer. To the initial solution, micro-liter additions of a solution that contained the dendrimer at the same concentration and the quencher at a known concentration were performed and the one-and twophoton excited fluorescence spectra were repeatedly acquired after each micro-liter addition. Stern-Volmer plots of the twophoton excited measurements were made with the acquired data.
From a known estimate of the maximum value of the bimolecular quenching constant controlled by the diffusion [22], the following relation was used to determine the contribution from dynamic quenching.

$$
Z=k_{0}[Q]
$$

where $Z$ is the collisional frequency of a chromophores, $k_{0}$ is the diffusion controlled bimolecular rate constant and $[Q]$, the concentration of the quencher. Simple estimation of the TNTdiffusion-controlled quenching at the quencher concentration of $10^{-3} \mathrm{M}$ using this formula [22] gives the maximum value of collision time about $100 \mathrm{~ns}$ whereas the experimental timescale of the dynamic quenching is in the picosecond range from time-resolved measurement studies.

\subsection{Time-resolved fluorescence measurements}

The sample solution was excited with frequency-doubled light from a mode-locked Ti-sapphire laser (Tsunami, Spectra Physics). This produces pulses of approximately $100 \mathrm{fs}$ duration in a wavelength range of $385-430 \mathrm{~nm}$. The polarization of the excitation beam for the anisotropy measurements was controlled with a Berek compensator. The horizontally polarized fluorescence emitted from the sample was up-converted in a nonlinear crystal of $\beta$-barium borate using a pump beam at about $800 \mathrm{~nm}$ that was first passed through a delay line. This system acts as an optical gate and enables the fluorescence to be resolved temporarily with a time resolution of about $200 \mathrm{fs}$ (pump-excitation 800/400 nm cross correlation function had a fwhm of $180 \mathrm{fs}$ ) [23-25]. Spectral resolution was achieved by dispersing the up-converted light in a monochromator and detecting it by using a photomultiplier tube (Hamamatsu R1527P).

\section{Results and discussion}

Previous work has shown that the assemblies of strong twophoton absorbing chromophores in dendritic structures have resulted in large $\delta_{\text {TPA }}[26,27]$. Our G-4 system possesses 96 two-photon absorbing chromophores on the near spherical surface of the dendrimer. These chromophores are quasi1D quadrupolar systems assembled from the grafting of donor moieties by the use of conjugated spacers onto a fluorenyl core [21]. Such chromophores present in G4 have been reported by our group previously to exhibit very large TPA cross-sections per entire dendrimer molecule reaching $\sim 56000 \mathrm{GM}$ (1 GM $=10^{-50} \mathrm{~cm}^{4} \mathrm{~s} /$ photon) [21]. This is much higher than that for sensory polymers previously investigated [28], either per entire molecule ( $~ 56000 \mathrm{GM}$ versus $\sim 2300 \mathrm{GM}$ ) or per one repeat unit (582 GM versus 54 GM, one order of magnitude higher) [28] (see footnote 5). Linear polymers are normally poly-dispersed with a large number of different chain lengths, thus making detailed structure-property relationships difficult to establish. Also in polymers, aggregation of the chains is possible. Molecular aggregation has shown to have detrimental effects on the twophoton properties [29]. This condition is avoided in dendrimers 


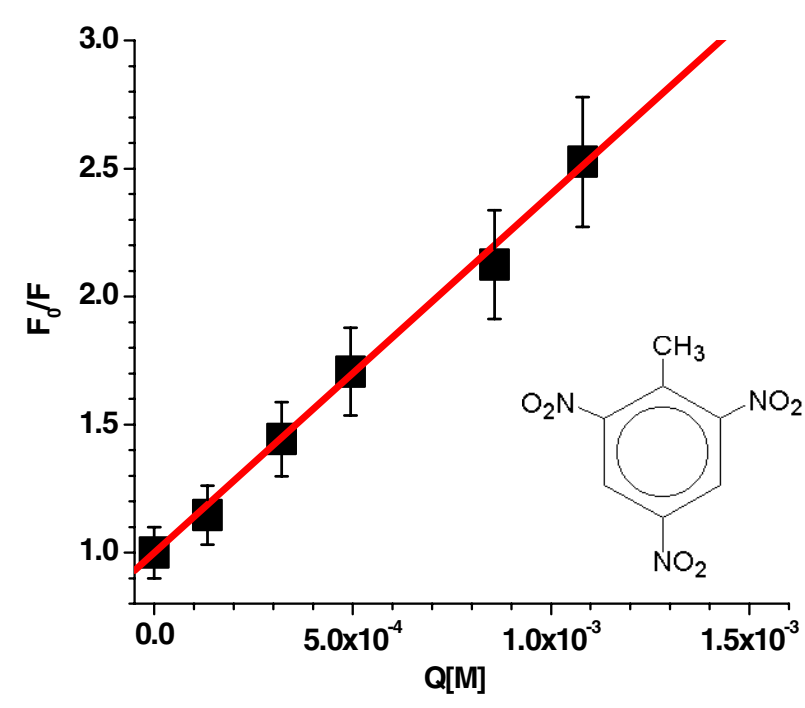

Figure 2. Stern-Volmer plot. Two-photon excited fluorescence quenching of dendrimer G4 by TNT. It is seen that the scattering of the data points characterizing the signal-to-noise ratio of two-photon approach with relatively small average laser intensity of a few milliwatts remains quite small and comparable with similar dependences obtained using one-photon excitation [30].

due to the confinement approach based on covalent grafting on the dendritic structure [21].

Fluorescence quenching by TNT were carried out by micro-titration in solution. In these experiments we found that the fluorescence of the dendrimers G4 is very sensitive to the presence of TNT independently of the excitation route (one- or two-photon). In addition to the sensitivity of G4, measurable signals of the quenched fluorescence were also observed with the smaller generations G1-G3, which possess 12, 24 and 48 chromophores respectively. Because the sensitivity increases with the generation number the complete quenching response of G4 is reported here. In order to quantify the quenching efficiency we used the Stern-Volmer relation [22]

$$
I_{0} / I=1+K_{\mathrm{SV}}[M]
$$

Stern-Volmer plot for the dendrimer G-4 under twophoton excitation $(800 \mathrm{~nm})$ in response to TNT is shown in figure 2. The Stern-Volmer quenching constant for this dendrimer $K_{\mathrm{SV}}$ was found to be $\sim 1400 \mathrm{M}^{-1}$. Comparing the Stern-Volmer factor obtained for the G4-dendrimer in solution, with those obtained under similar conditions for the various amplifying polymers which were used for TNT detection [30] one can see that the dendrimer's S-V-factor $K_{\mathrm{SV}}$ is systematically higher.

We have also directly compared the Stern-Volmer profile for the dendrimer with that for the pentipticene-containing polymer highly sensitive to TNT [8] and found the $K_{\mathrm{SV}}$ in toluene for the dendrimer is twice as large as that for polymer under the same conditions (1400 $\mathrm{M}^{-1}$ versus $\left.702 \mathrm{M}^{-1}\right)$ [28] (see footnote 5). Therefore, along with the much higher twophoton absorption cross-section mentioned above [28] (see footnote 5), the dendrimer in solution demonstrated superior quenching response to TNT than polymers. Regarding stability of the system, we have determined that the dendritic systems are very stable structures to the environment, specifically. Absorption spectra of the samples taken after a span of one year show no change, rendering them a good shelf life for sensingbased applications

To better understand the origin of the enhanced sensing performance of this new dendrimer system we have investigated the quenching mechanism in more detail. The linear Stern-Volmer profile usually indicates either purely static or dynamic quenching while the simultaneous presence of static and dynamic quenching is expected to result in the superlinear Stern-Volmer profile [22]. Previous studies on conjugated polymers with amplified quenching in solutions have shown that the one-photon excited fluorescence quenching by nitro-aromatics followed a superlinear SternVolmer relation indicating the presence of both static and diffusion controlled dynamic quenching [22]. In some cases the saturation of the static quenching due to 'loose' aggregation can lead to the linear $\mathrm{S}-\mathrm{V}$ profile even in the presence of dynamic component as it has been recently reported for the polyelectrolyte quenching [16]. In order to shed more light on the nitro-aromatic quenching mechanism in these new dendrimers we have performed femtosecond timeresolved fluorescence upconversion studies on the dendrimer generation G3 under one and two-photon excitations. From previous studies on dendrimers by our group, it was observed that the dendrimers can possess excellent exciton transport properties [31] implying the possibility of amplified quenching in these systems. Figure 3 displays the difference between fluorescence dynamics for dendrimer sample with TNT (concentration: $\left.5 \times 10^{-3} \mathrm{M}\right) F_{\mathrm{q}}(t)$ and that for pure dendrimer solution $F_{0}(t)$. The ratio (quenching factor) $Q(t)=$ $F_{\mathrm{q}}(t) / F_{0}(t)$ represents the fraction of quenched excitations at a given delay time $t$. It is seen from this figure that $\sim 10 \%$ of fluorescence is quenched within the time resolution of the upconversion setup $\sim 100$ fs. Taking into account the absence of any measurable fast dynamics for $Q(t)$ in the vicinity of instrument response function (inset of figure 3(b)) this fraction of quenching can be considered as instantaneous or true static. However the major part of the fluorescence quenching by TNT occurs on the timescale $\sim 150 \mathrm{ps}$ as shown in figure $3(\mathrm{~b})$, and should be considered as a dynamic quenching. The best fit of the time-resolved quenching factor $Q(t)$ showed multi-exponential decay with time components of $1.5,17$ and 160 ps. It is also seen from figure 3(b) that static and dynamic quenching components taken together account for the fluorescence quenching by $\sim 50 \%$ at long delays which is in excellent agreement with steady state measurements showed the same total amount of quenching at this TNT concentration.

Simple estimation of the TNT-diffusion-controlled quenching at the quencher concentration at $10^{-3} \mathrm{M}$ using this formula gives the maximum value of collision time of about $100 \mathrm{~ns}$ [22] whereas the experimental timescale of the dynamic quenching is in the picosecond range from time-resolved measurement studies shown in figure 3 . This proves that in our case the contribution from the dynamic quenching associated with the diffusion of TNT in solution is negligible. For this dendrimer the 
(a)

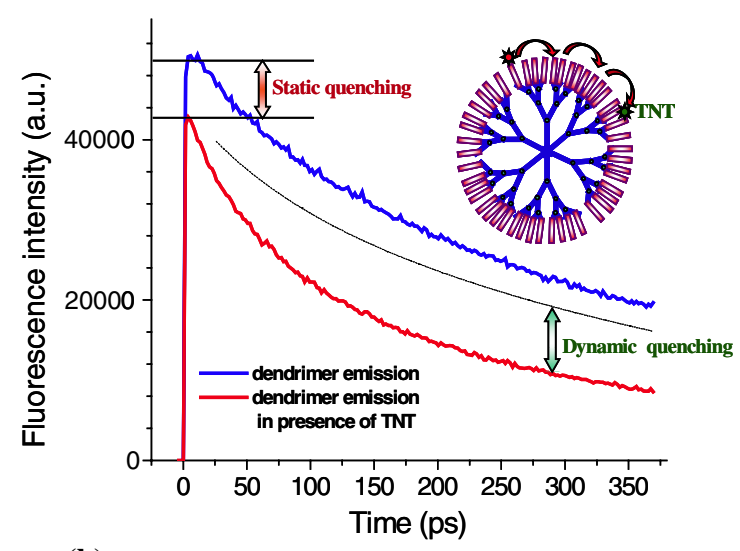

(b)

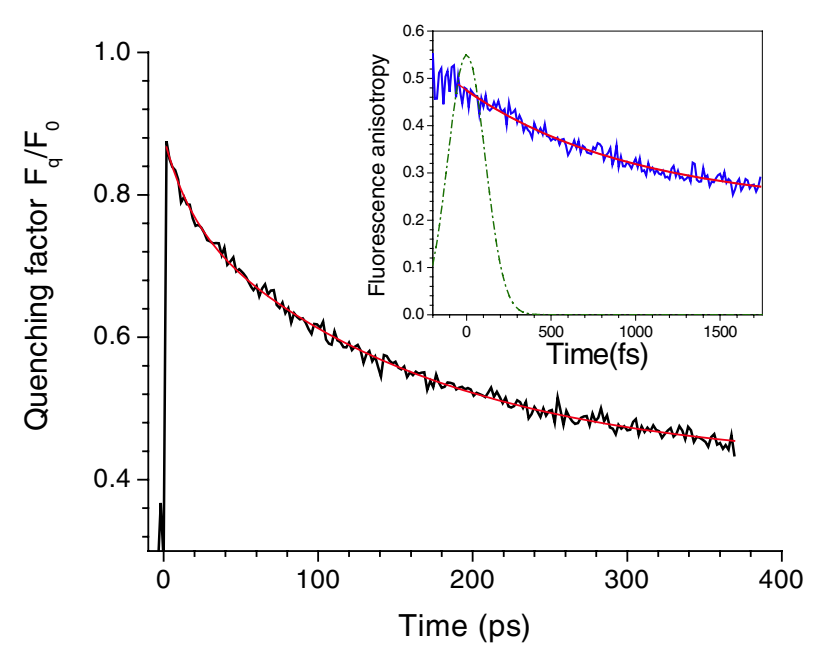

Figure 3. Time-resolved measurements. (a) Difference in fluorescence dynamics shown, with an initial fractional static contribution, followed by a major dynamic contribution. Inset shows travel of exciton sampled by TNT over spherical dendrimer surface. (b) Quenching factor as a function of time shows that the drop in fluorescence by a factor due to both static and dynamic contributions occurs at long delay times. Inset shows instrument response.

fluorescence quenching is in major part a fast dynamic process on a picosecond timescale, which can result from the fast exciton migration on the dendrimer surface from the excitation site to the site associated with TNT-chromophore complex. The exciton migration over the dendrimer spherical surface can be monitored using time-resolved fluorescence anisotropy measurements. Exciton migration over the surface of the dendrimer leads to the reorientation of the emitting dipole and fluorescence depolarization. The fluorescence anisotropy decay thus reflects the timescale of the exciton migration over the dendrimer surface [31]. As shown in the inset of figure 3(b) the initial anisotropy is high, implying that the excitation is initially localized on one chromophore [31]. The anisotropy decay gave a time component of $1.1 \mathrm{ps}$ for the initial decay from the initial anisotropy of 0.5 for two-photon excitation mode, and longer time components of 16 and 98 ps. These time components describing the exciton migration over the dendrimer surface are in the same range as those observed for the dynamic quenching, thus proving that the exciton migration over the dendrimer surface is the main contributor to the observed dynamic fluorescence quenching. This validates the efficient amplified quenching mechanism associated with exciton migration over dendrimer surface as a main quenching mechanism. Our results also indicate that the migration over quasi-two-dimensional dendrimer surface results in more efficient amplified quenching than that along the one-dimensional polymer chains ('molecular wires').

\section{Summary}

In conclusion, we have demonstrated a valuable approach of two-photon excitation route for TNT sensors based on the fluorescence quenching. We have synthesized and investigated new dendritic materials as promising two-photon sensors for TNT detection. These materials possess the exceptional combination of large $\delta_{\text {TPA }}$ with efficient amplified fluorescence quenching by TNT. It is also worth noting that two-photon excitation can potentially provide even higher selectivity by way of excitation of the specific two-photon excited states of particular symmetry [32] which may be more susceptible to quenching by the analyte. The opportunity to performing long range remote sensing applications with femtosecond IR pulses via the two-photon absorption method is thus an attractive alternative to conventional explosives detection. The employment of two-photon excitation for stand-off detection therefore proves to be a significant step forward in the field of fluorescence chemical sensing.

\section{Acknowledgments}

We would like to thank the Army Research Office (Materials) and the National Science Foundation (Polymers) for support of this research.

\section{References}

[1] DeVolpi A and Rhodes E A 1992 Science 25814

[2] Wang D, Gong X, Heeger P S, Rininsland F, Bazan G C and Heeger A J 2002 Proc. Natl Acad. Sci. USA 9949

[3] Cumming C J, Aker C, Fisher M, Fox M, La Grone M J, Reust D, Rockley M G, Swager T M, Towers E and Williams V 2001 IEEE Trans. Geosci. Remote Sens. 391119

[4] Swager T M 1998 Acc. Chem. Res. 31201

[5] Sohn H, Sailor M J, Magde D and Trogler W C 2003 J. Am. Chem. Soc. 1253821

[6] Liu Y, Mills R C, Boncella J M and Schanze K S 2001 Langmuir 177452

[7] McQuade D T, Pullen A E and Swager T M 2000 Chem. Rev. 1002537

[8] Yang J-S and Swager T M 1998 J. Am. Chem. Soc. 12011864

[9] Tao S, Li G and Zhu H 2006 J. Mater. Chem. 164521

[10] Naddo T et al 2007 J. Am. Chem. Soc. 1296978

[11] Cumpston B H et al 1999 Nature 39851

[12] Albota M et al 1998 Science 2811653

[13] Bhawalkar J D, Kumar N D, Zhao C F and Prasad P N 1999 J. Clin. Laser Med. Surg. 15201

[14] Goodson T III 2005 Acc. Chem. Res. 3899 
[15] Varnavski O, Yan X, Mongin O, Blanchard-Desce M and Goodson T III 2007 J. Phys. Chem. C 111149

[16] Müller J G, Atas E, Tan C, Schanze K S and Kleiman V D 2006 J. Am. Chem. Soc. 1284007

[17] Scholes G D and Rumbles G 2006 Nat. Mater. 5683

[18] Devadoss C, Bharathi P and Moore J S 1996 J. Am. Chem. Soc. 1189635

[19] Bosman A W, Janssen H M and Meijer E W 1999 Chem. Rev. 991665

[20] Varnavski O et al 2002 J. Chem. Phys. 1168893

[21] Mongin O et al 2006 Chem. Commun. 915

[22] Lakowicz J 1999 Principles of Fluorescence Spectroscopy 2nd edn (New York: Kluwer Academic/Plenum) pp 239-43

[23] Wang Y, He G S, Prasad P N and Goodson T III 2005 J. Am. Chem. Soc. 12710128
[24] Varnavski O, Yan Z, Mongin O, Blanchard-Desce M and Goodson T III 2007 J. Phys. Chem. C 111149

[25] Varnavski O et al 2002 J. Chem. Phys. 1168893

[26] Drobizhev M, Karotki A, Rebane A and Spangler C W 2001 Opt. Lett. 261081

[27] Mongin O, Brunel J, Porres̀ L and Blanchard-Desce M 2003 Tetrahedron Lett. $\mathbf{4 4} 2813$

[28] Narayanan A, Varnavski O P, Swager T M and Goodson T III 2007 J. Phys. Chem. C submitted

[29] Woo H Y et al 2005 J. Am. Chem. Soc. 12714721

[30] Zhao D and Swager T M 2005 Macromolecules 389377

[31] Varnavski O P et al 2002 J. Am. Chem. Soc. 1241736

[32] Frolov S V, Bao Z, Wohlgenannt M and Vardeny Z V 2000 Phys. Rev. Lett. 852196 